\title{
Frequently Asked Questions about Maintenance of Certification for Family Physicians
}

Introduction: The specialty of Family Practice has a long tradition as a leader in the field of recertification. It was the first specialty Board to issue time-limited certificates in 1970 and to require mandatory recertification every seven years. The founders of the American Board of Family Practice (ABFP) had the foresight to create a process which has served the specialty well for the past 34 years. Recently, the American Board of Medical Specialties (ABMS), sensing growing and repeated threats from outside of medicine, determined that it was necessary to develop a program that would assure the American public that ABMS specialists within every discipline met the highest standards of accountability. They mandated that this program continuously measure physician competencies and that this "should be demonstrated throughout the physician's career by evidence of lifelong learning and ongoing improvement of practice". More importantly, they designed a process called Maintenance of Certification (MOC), in which every ABMS board-certified specialist in the United States would be expected to participate.

The American Board of Family Practice has been busy developing a unique program for family physicians within the framework specified by the ABMS. Below find the answers to some frequently asked questions about Maintenance of Certification for Family Physicians (MC-FP).

Q What is "Maintenance of Certification" and what is expected of each Diplomate?

A Maintenance of Certification is a program mandated by the ABMS with the explicit purpose of providing a mechanism for measuring, in a continuous fashion, the ongoing competencies of practicing physicians in every specialty. These competencies are medical knowledge, patient care, interpersonal and communication skills, professionalism, practicebased learning and improvement, and systems-based practice.

The ABMS has developed a framework, which consists of four unique components that will measure the six competencies on a continuous basis. These four components include: 1) Evidence of Professionalism; 2) Evidence of Self-assessment and Lifelong Learning; 3) Evidence of Cognitive Expertise; and 4) Evidence of Assessment of Performance in Practice. Each Member Board of the ABMS will be required to develop specific mechanisms for assessing its Diplomates in each of these four areas.

\section{When will the ABFP launch its Maintenance of Certification program?}

A The ABFP will phase in its MC-FP program beginning January 1, 2004 and extending to 2010.
The first group of individuals who enter the MC-FP program will be those who either certify or recertify in Family Practice in 2003.

\section{Q Why is the American Board of Family Practice (ABFP) instituting this now?}

A The ABMS has requested that the ABFP, as well as all member ABMS boards, submit their plans for institution of the first three parts of this program (excluding Part 4 - evidence of performance in practice) by July 1, 2003 with the expectation that all member boards will begin their MOC program shortly thereafter. The ABMS will require that all member boards submit their plans for Part 4 by December 31, 2003.

Q

What is meant by the phrase, "Refining the Paradigm - the Transition from Recertification to Maintenance of Certification"?

A For close to 30 years, we have been utilizing parts of each of the four components of the MOC process in the recertification process. Therefore, unlike other specialists who will be required to do this having never done it before, our diplomates will at least have had some experience with the basic aspects of the process. By way of example, we have required a full 
and unrestricted license in every state in which the Diplomate practices; 300 hours of Continuing Medical Education (CME); a recertification examination; and the Computerized Office Record Review (CORR). Each of these components bears striking resemblance to the four components of MOC as outlined by the ABMS. Each of these four recertification components needs only to be modified slightly to meet the new requirements for MOC established by the ABMS. That's why we are calling this "refining the paradigm" rather than making a paradigm shift to an entirely new and different model.

\section{Q What does the successful launch of the MC-FP program mean for all Family Practice Diplomates and the Specialty?}

A This will provide the mechanism by which we can continuously assess the competencies of the practicing Family Physician and do so in a scientifically reliable and valid manner. The ABMS is working diligently to see that the federal government, third party payers, and state licensing boards recognize MOC and use this process to replace current or future requirements that these agencies may impose on the practicing physician, thereby relieving the busy, practicing family physician of tremendously burdensome tasks. By taking the lead in this area, we envision the MC-FP process as a value added component of the physician's practice. Not only could this program potentially avoid time-consuming tasks such as quality assurance audits by third-party payers or re-licensing exams by state licensing agencies, but it will also assure that family physicians stay abreast of the state of the art in our specialty, and more importantly, apply it to the care of their patients.

\section{Q Whom does it affect?}

A This will affect every single Diplomate of the ABFP, although the program will be phased in, so it won't affect everyone at once. From this point forward those who will either certify, or who will recertify under the old process, will immediately enter into the MC-FP program. They will be issued a time-limited certificate good for seven years, as has always been done, and enter the seven year MC-FP cycle. In order to maintain their certificate, they need to successfully complete all four components of the MC-FP process during that seven year period.

\section{$Q$ What are those components?}

A 1) They need to demonstrate evidence of professionalism continuously throughout the cycle.
They will need to continue to possess a full, unrestricted license in all states in which they maintain a license. The ABMS is currently developing a patient satisfaction instrument that it envisions will be used by Boards to measure another aspect of professionalism. This will be available in 2004 or 2005 . In this regard, it is important to note that MC-FP is an evolving program. We will be starting out with one set of mechanisms to measure competencies in each of the four components. However, as the science to measure these competencies advances, we will be incorporating new tools, and replacing old ones with more advanced ones.

2) Evidence of self-assessment and lifelong learning. We will offer a variety of clinical self-assessment modules from which a Diplomate will be able to select one to perform per year. The first two that will be available next year will be Diabetes and Hypertension. Asthma and Coronary Artery Disease will be added in 2005. We are working closely with the American Academy of Family Physicians (AAFP) and other stakeholders within our discipline to coordinate the roll out of our self-assessment modules with their continuing medical education initiatives. Each clinical self-assessment module will consist of two parts. Part A will be an assessment of the physician's knowledge with respect to the disease domain. Part B will be an assessment of the Diplomate's ability to apply that knowledge in a clinical setting. We will use our patient simulation technology to assess this. Both Parts A and B of the clinical self-assessment modules will be web delivered. Diplomates will be able to take them at any time and at any place where they have computer access. They can take these as many times as necessary to pass each part. Part A must be successfully completed before moving on to Part B. Candidates must successfully complete six clinical self-assessment modules during the seven year MC-FP cycle.

If Diplomates have a Certificate of Added Qualification (CAQ) in Adolescent Medicine, Geriatric Medicine or Sports Medicine, one of the six clinical self-assessment modules must be taken within the discipline of their CAQ. If they hold two CAQs, one module in the area of each CAQ must be taken plus four other modules for a total of six self-assessment modules completed during the seven year MCFP cycle.

The current 300 hour CME requirement will remain. However, the time actually spent by the Diplomate in completing the self-assessment modules will be credited toward this requirement.

3) Evidence of cognitive expertise. This will be measured by examination. The examination will be offered in the 6 th or 7 th year of the MC-FP cycle. We are transitioning into delivering the examination 
entirely on the computer. Next year we will offer the examination at a number of paper and pencil sites as well as 200 computer-based testing centers throughout the United States. In 2005, the examination will be delivered entirely on computer over multiple dates at the 200 computer-based testing centers. It is estimated that $80 \%$ of our Diplomates will be within a one-hour drive of one of these testing centers.

4) Evidence of assessment of performance in practice. We will introduce this in late 2004. Initially, this will be done by modifying our current Computerized Office Record Review process. The Diplomate will choose a specific disease domain and will select 10 patient charts for audit. This audit will be done via the web at the physician's convenience. The physician will abstract information from the chart, which will be transmitted to us. This information will be measured against evidence-based quality indicators and the physician will be given feedback on their performance. Based on their performance, they will then go to a section on our website to develop a quality improvement plan which will be individually tailored. At a later point in time during the MC-FP cycle, the Diplomate will be asked to repeat the audit so that we can determine whether in fact the quality improvement program that they have developed has resulted in improvement in this aspect of their practice.

One assessment of performance in practice module must be completed during the seven-year MC-FP cycle. We are working with the AAFP to make certain that the quality improvement initiatives that they are currently designing for their members will be suitable for satisfying this component of MCFP. The actual time spent completing this module may be credited against the current 300 hour CME requirement.

\section{Will it cost me more to participate in MC-FP than in the recertification process?}

A We are making every effort to make participation in MC-FP efficient and convenient for the Diplomate. When fully operational in 2005 , we believe that the costs associated with making this program web-based and computer delivered will be more than offset by the savings realized by avoiding travel, hotel costs and lost opportunity income from practice associated with taking the recertification examination at a limited number of written test centers on a single day.

\section{Q How do I prepare for MC-FP?}

A We are collaborating with the AAFP, as well as all stakeholders within our specialty, to make certain that they are fully aware of how we plan to proceed with MC-FP. We would expect that they will develop unique Continuing Medical Education (CME) programs which will be targeted at helping Family Physicians participate in the MC-FP process.

\section{Q Do I have more than one opportunity to take various components of $M C-F P$ ?}

A Of course, as in the past, one will need to maintain a current and unrestricted license throughout the MC-FP cycle. With respect to Part 2, self-assessment and lifelong learning, the Diplomate will be able to take the clinical self-assessment modules as many times as necessary to pass. The cognitive examination will be given multiple times per year, and therefore, there is the possibility that if the candidate took the exam in the sixth year after satisfying all other components of MC-FP and failed early in the year, they could take it later in the year in an attempt to successfully complete the examination. If they failed the repeat exam, they could take the exam again early in the seventh year with the possibility of taking the exam again later in the seventh year if they failed. So theoretically, the Diplomate could take the exam as many as four times in an attempt to pass within the MC-FP cycle.

\section{Q I bave a questions regarding Process $B$. I am one of many Family Physicians who spent most of their time in leadership roles while practicing part-time. There are elements of the new program that do not fit us Process B-types very well. How will the MC-FP be achievable for Process $B$ candidates?}

A You will be able to maintain your certification if you do not see patients or do not see patients in continuity. We will create a unique modules for Part IV (e.g., patient safety, systems based practice) that will allow non-practicing physicians the opportunity to complete this part of MC-FP. 